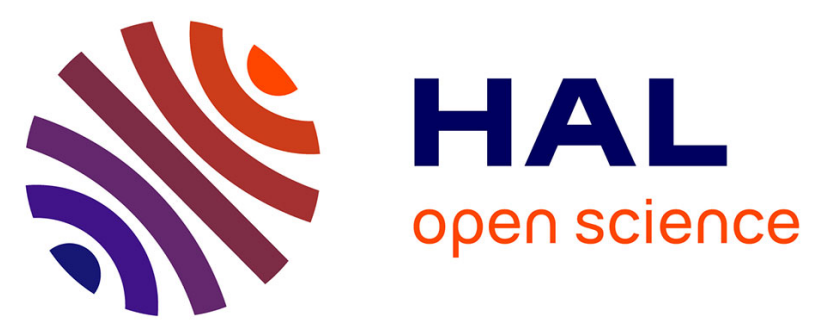

\title{
METAL-NON METAL TRANSITIONS IN TRANSITION METAL COMPOUNDS.THE ELECTRONIC PHASE TRANSITIONS IN FeS AND $\mathrm{NiS}$
}

\author{
J. Coey, H. Roux-Buisson, R. Brusetti
}

\section{To cite this version:}

J. Coey, H. Roux-Buisson, R. Brusetti. METAL-NON METAL TRANSITIONS IN TRANSITION METAL COMPOUNDS.THE ELECTRONIC PHASE TRANSITIONS IN FeS AND NiS. Journal de Physique Colloques, 1976, 37 (C4), pp.C4-1-C4-10. 10.1051/jphyscol:1976401 . jpa-00216515

\section{HAL Id: jpa-00216515 https://hal.science/jpa-00216515}

Submitted on 1 Jan 1976

HAL is a multi-disciplinary open access archive for the deposit and dissemination of scientific research documents, whether they are published or not. The documents may come from teaching and research institutions in France or abroad, or from public or private research centers.
L'archive ouverte pluridisciplinaire HAL, est destinée au dépôt et à la diffusion de documents scientifiques de niveau recherche, publiés ou non, émanant des établissements d'enseignement et de recherche français ou étrangers, des laboratoires publics ou privés. 


\title{
THE ELECTRONIC PHASE TRANSITIONS IN FeS AND NiS
}

\author{
J. M. D. COEY, H. ROUX-BUISSON and R. BRUSETTI \\ Groupe des Transitions de Phases, C. N. R. S., \\ B. P. 166, 38042 Grenoble Cedex, France
}

\begin{abstract}
Résumé. - A $T_{\alpha}=415 \mathrm{~K}$ et $T_{\mathrm{t}}=265 \mathrm{~K} \mathrm{FeS}$ et NiS présentent respectivement une transition de phase de premier ordre accompagnée d'un changement de structure électronique. La température de transition de NiS dépend fortement du taux de lacunes en nickel. Un grand nombre de données expérimentales montrent que dans la phase basse température NiS est un semi-métal antiferromagnétique délocalisé et que dans la phase haute température, c'est un métal paramagnétique de Pauli faiblement corrélé. Un nombre plus restreint d'expériences suggèrent que FeS dans la phase basse température, est un semiconducteur antiferromagnétique dégénéré et que dans la phase haute température, c'est un mauvais métal antiferromagnétique où le fer garde son moment. Dans les deux composés, le réseau participe fortement au changement d'entropie à la transition. Dans NiS, la contribution électronique représente le quart de l'entropie totale de la transition, la densité d'état au niveau de Fermi étant alors multipliée par huit. En revanche, dans FeS, la contribution magnétique à l'entropie de la transition est importante en raison de l'affaiblissement de l'échange à $T_{\alpha} . N_{0,9} S$ est un métal paramagnétique faiblement corrélé à hautes températures, des impuretés de fer y présentent un comportement de type Kondo jusqu'à des concentrations de l'ordre de $1 \%$, à plus fortes concentrations le fer se comporte comme un verre de spin ou un antiferromagnétique dilué. Un diagramme de phases préliminaire montre trois régions pour le système $\left(\mathrm{Ni}_{1-x} \mathrm{Fe}_{x}\right)_{1-\delta} \mathrm{S}$.
\end{abstract}

I. $\mathrm{Ni}$ et $\mathrm{Fe}$ sont magnétiquement ordonnés,

II. Ni et Fe sont respectivement paramagnétiques de Pauli et de Curie-Weiss ou de type Kondo,

III. Fe est ordonné magnétiquement, Ni est paramagnétique de Pauli.

\begin{abstract}
FeS and NiS show first order phase transitions with a change in electronic structure at $T_{\alpha}=415 \mathrm{~K}$ and $T_{\mathrm{t}}=265 \mathrm{~K}$ respectively, the latter depending sensitively on non-stoichiometry in the form of nickel vacancies. Extensive experimental data on NiS is reviewed which shows that the low-temperature phase is a semimetallic itinerant-electron antiferromagnet and the high temperature phase a weakly-correlated Pauli paramagnetic metal. Less complete data on FeS suggests the low temperature phase is a degenerate antiferromagnetic semiconductor and the hightemperature phase an antiferromagnetic poor metal where the iron retains its local moment. In both compounds the increase in lattice entropy is an important contribution to the increase in entropy on heating through the transitions. In NiS, the electronic contribution provides approximately one fourth of the total entropy of transition due to the eight fold increase in density of states at the Fermi level at $T_{\mathrm{t}}$ whereas in $\mathrm{FeS}$, there is a large magnetic contribution due to weakening of the exchange at $T_{\alpha} . \mathrm{Ni}_{0.95} \mathrm{~S}$ is a weakly-correlated paramagnetic metal at all temperatures and iron impurities show Kondo-like behaviour at a concentration below $1 \%$, and dilute antiferromagnetic or spin-glass-like order at higher concentrations. A preliminary $T: x: \delta$ phase diagram for the system $\left(\mathrm{Ni}_{1-x} \mathrm{Fe}_{x}\right)_{1-\delta} \mathrm{S}$ shows three distinct regions :
\end{abstract}

I. antiferromagnetic order of both $\mathrm{Ni}$ and $\mathrm{Fe}$,

II. paramagnetic $\mathrm{Ni}$ and $\mathrm{Fe}, \mathrm{Ni}$ being a Pauli paramagnet and $\mathrm{Fe}$ a Curie-Weiss paramagnet or Kondo-like,

III. Fe magnetically ordered while Ni remains Pauli paramagnetic.

1. Introduction. - Many compounds of the transition metals with the heavier elements of the Vth and VIth subgroups possess the NiAs structure. Although the cations are formally di- or trivalent, the actual charge transfer from the metal is rather small in the chalcogenide or pnictide, and the bonding tends to be covalent or metallic rather than ionic.

The NiAs structure is illustrated in figure 1. It consists of alternate hexagonal close-packed layers of nickel and arsenic with a stacking sequence ABAC... All the metal planes are indentical, and the octahedra of arsenic surrounding the nickel share faces, perpendicular to the hexagonal $c$-axis. The structure can often tolerate wide variations in stoichiometry, usually in the form of metal deficiency. Even at the stoichiometric composition, large numbers of vacancies may occur on both sublattices [1].

Several NiAs-structure compounds exhibit phase transitions where the electronic structure is abruptly modified as a function of pressure, temperature or stoichiometry. The consequences for the resistivity are less dramatic than in transition-metal oxides, yet the rich variety of electronic properties invites detailed study and classification nonetheless. Here, we consider two such compounds, FeS and NiS, which present many points of similarity. Both are golden in colour. 


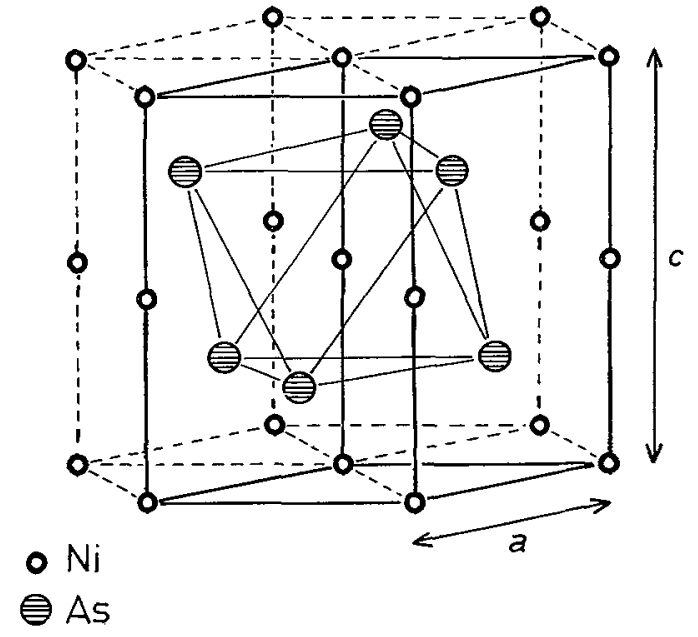

FIG. 1. - The NiAs structure.

The chemical shifts of core lines measured by X-ray photoemission spectroscopy (ESCA) indicate a charge transfer of only 0.4 electrons as compared with the elements in both compounds. Spectra for binding energies close to the Fermi level are shown in figure 2. The broad emission extending to $B \simeq 6 \mathrm{eV}$ is attributed to sulphur $3 p$ electrons, whereas the peak at $E_{\mathrm{F}}$ with a width $\simeq 2 \mathrm{eV}$ in NiS is attributed to nickel $3 \mathrm{~d}$ electrons. The structure due to iron $3 \mathrm{~d}$ electrons in

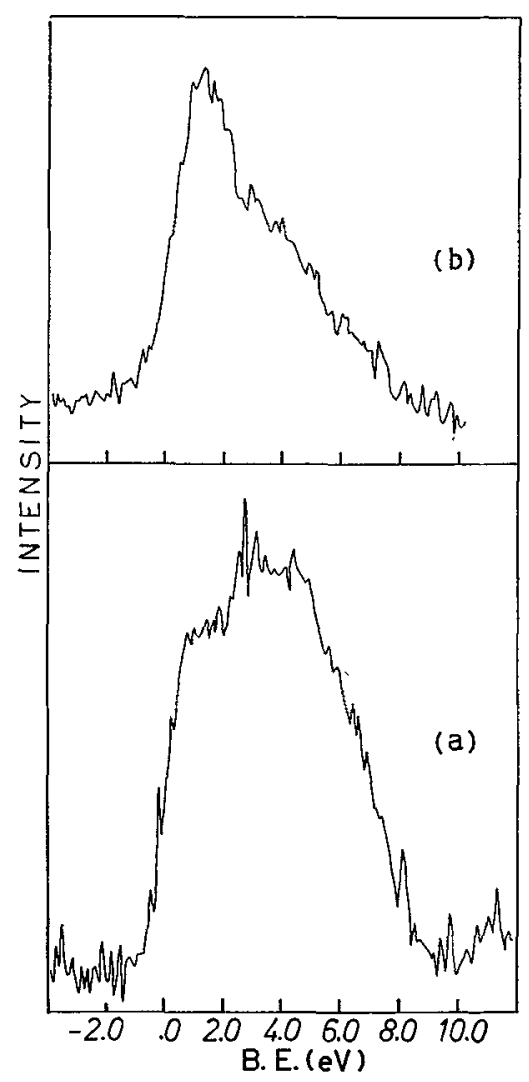

Fig. 2. - ESCA spectra of a) FeS and $b$ ) NiS close to the Fermi energy. The instrumental resolution is $0.7 \mathrm{eV}$.
FeS is less well-resolved, but it seems clear that the $3 \mathrm{~d}$ and $3 p$ bands overlap in energy in both cases. Each compound exhibits a first-order electronic phase transition as a function of increasing temperature with an entropy increase of $\simeq 5 \mathrm{~J} /$ mole $\mathrm{K}$. Although there is barely any change in the gross electronic structure as measured by $\mathrm{X}$-ray photoemission, the resistivity drops sharply, by as much as two orders of magnitude in stoichiometric samples $[2,3]$, and there are corresponding changes in the heat capacity, magnetic susceptibility, lattice parameters and thermoelectric power, all shown in figures 3 and 4 . In the low temperature phase of FeS the structure is a slight distortion [4] of the NiAs structure but in NiS there is no symmetry change [5]. For stoichiometric samples, the transition in NiS occurs on heating at $T_{t}=268 \mathrm{~K}$ [6] and that in $\mathrm{FeS}$ at $T_{\alpha}=424 \mathrm{~K}$ [3]. Fairly extensive references to

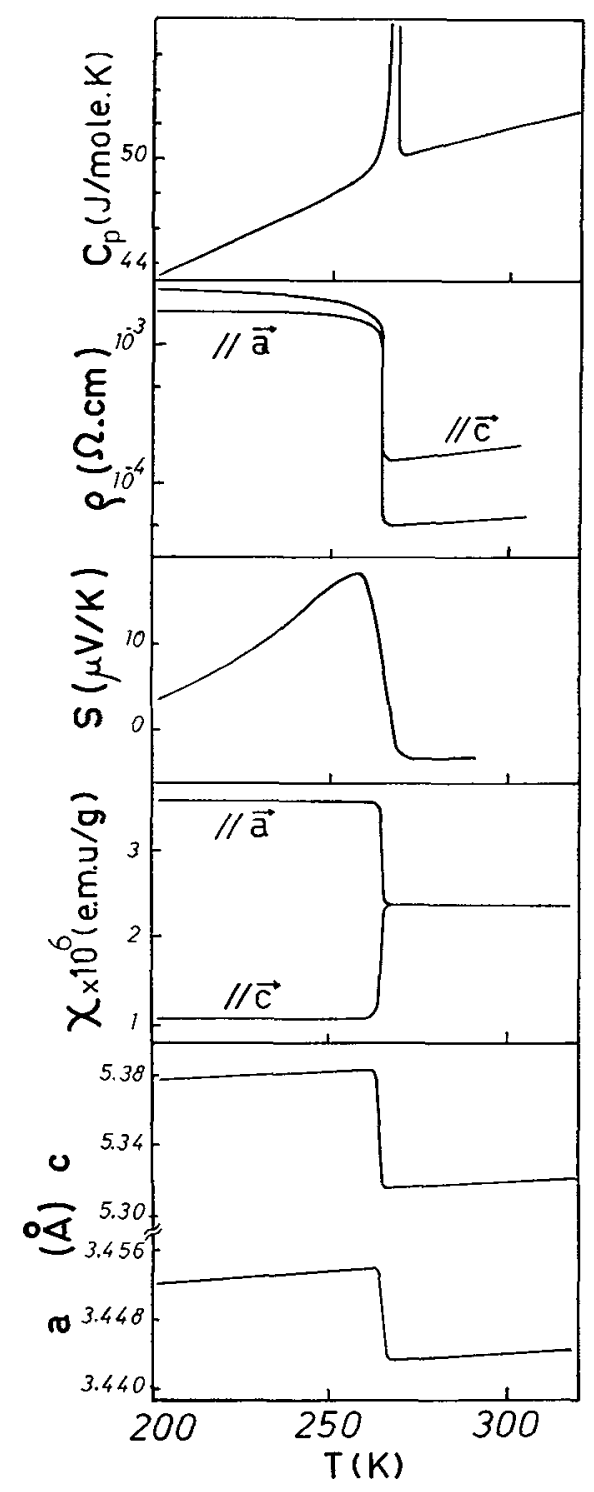

FrG. 3. - Changes in some physical parameters at the phase transition in NiS, a) specific heat, b) resistivity, c) thermoelectric power, $d$ ) magnetic susceptibility, e) lattice parameters from reference [9]. 


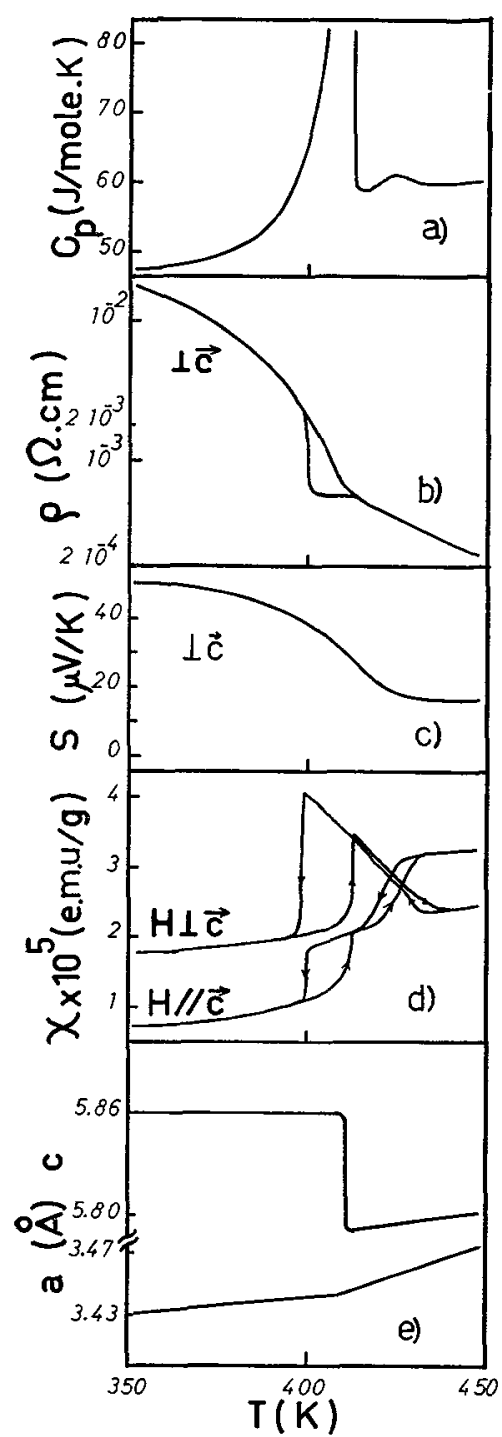

FIG. 4. - The same as figure 3, but for FeS, e) is from reference $[10]$.

the literature on NiS and FeS may be found in [7] and [29] or [8] and [44] respectively.

The plan in this paper is to begin by characterising the electronic structure of the two phases in each of the compounds, and then to attempt to understand the underlying reasons for the transitions. In spite of the many points of similarity, it will become evident that the electronic properties of $\mathrm{FeS}$ and $\mathrm{NiS}$ are really quite different. This difference is strikingly illustrated by the behaviour of iron impurities in metallic nickel sulphide described in the penultimate section. It is analogous to the difference between iron and nickel metal, the one having a well defined local moment, whereas in the other magnetism is itinerant.

2. NiS. - In nickel sulphide, the transition may be suppressed by external pressure, or by non-stoichiometry in the form of nickel vacancies, $\mathrm{Ni}_{1-\delta} \mathrm{S}$.
The $T_{\mathrm{t}}: \delta$ diagram is shown in figure 5. A pressure of $20 \mathrm{kbar}[5]$ has the same effect as $3 \%$ non-stoichiometry on the nickel sublattice - it stabilises the hightemperature phase down to zero temperature. In this

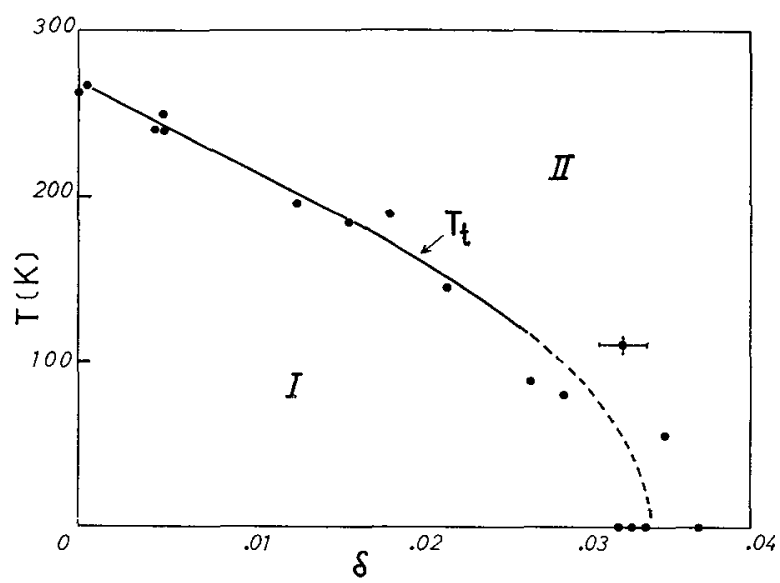

FIG. 5. - Variation of the first-order transition temperature with stoichiometry in $\mathrm{Ni}_{1-\delta} \mathrm{S}$. Phase $\mathrm{I}$ is an antiferromagnetic semimetal. Phase II is a Pauli-paramagnetic metal.

system, one can conveniently study both phases in a wide range of temperature by preparing crystals of slightly different composition.

Crystals up to several cubic centimeters in volume were prepared by slow cooling of the melt or by the Bridgmann method as described in [11]. A quench from $700 \mathrm{~K}$ was necessary to conserve the NiAs structure, which is actually metastable below about $650 \mathrm{~K}$. The truely stable form of NiS at low temperatures to which the NiAs form reverts if heated much above $300 \mathrm{~K}$ is millerite. Unlike the NiAs form we are considering here, millerite does not order magnetically [12] (see also Fig. 8).

2.1 High temperature phase $T>T_{\mathrm{t}}$. This is a normal nonmagnetic metal and there is no evidence for strong electronic correlations such as is found for $\mathrm{V}_{2} \mathrm{O}_{3}$ or $\mathrm{VO}_{2}$ [13]. The temperature dependence of the resistivity and magnetic susceptibility of $\mathrm{Ni}_{0.955} \mathrm{~S}$ are shown in figure 6. On a log-log plot, it appears that the temperature dependent part of the resistivity is proportional to $T$ above about $240 \mathrm{~K}$, to $T^{3}$ between 10 and $100 \mathrm{~K}$, and to $T^{2}$ only below $10 \mathrm{~K}$. The large residual resistivity, $10 \mu \Omega \mathrm{cm}$, is associated with the high concentration of crystal defects. The magnetic susceptibility is isotropic and virtually independent of temperature. Its magnitude $2 \times 10^{-4} \mathrm{emu} / \mathrm{mole}$ is typical of a Pauli paramagnet and the slight tendency for $\chi$ to increase with $T$ has already been interpreted as showing that the Fermi level lies near a minimum in the density of states $[14,15]$. The value of the Pauli susceptibility, corrected for core-electron diamagnetism, gives a density of states at the Fermi level of 7.7 states/eV/ molecule. On a free electron model, this corresponds 


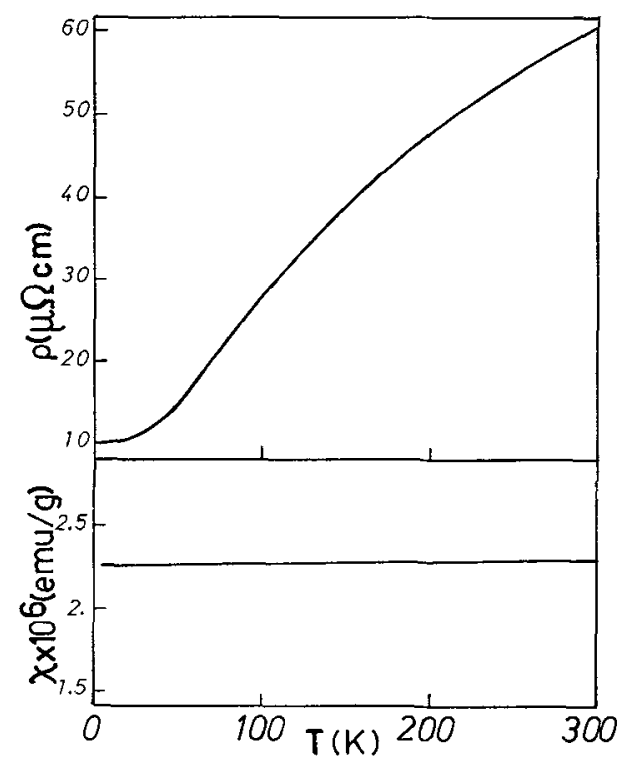

Frg. 6. - Temperature dependence of $a$ ) resistivity and $b$ ) susceptibility of $\mathrm{Ni}_{0.955} \mathrm{~S}$.

to $E_{\mathrm{F}}=0.4 \mathrm{eV}$. The Seebeck coefficient is typically metallic, small and proportional to temperature above $T_{\mathrm{t}}$ in figure $3 c$. Using the formula [16]

$$
S \simeq \frac{\pi^{2} k^{2} T}{2 e} \frac{1}{E_{\mathrm{F}}}
$$

we deduce $E_{\mathrm{F}}=1.0 \mathrm{eV}$. Finally, the electronic specific heat coefficient $\gamma$ is found to be $6 \mathrm{~mJ} / \mathrm{mole} \mathrm{K}^{2}$ in the metallic phase of NiS (Fig. 7). The corresponding

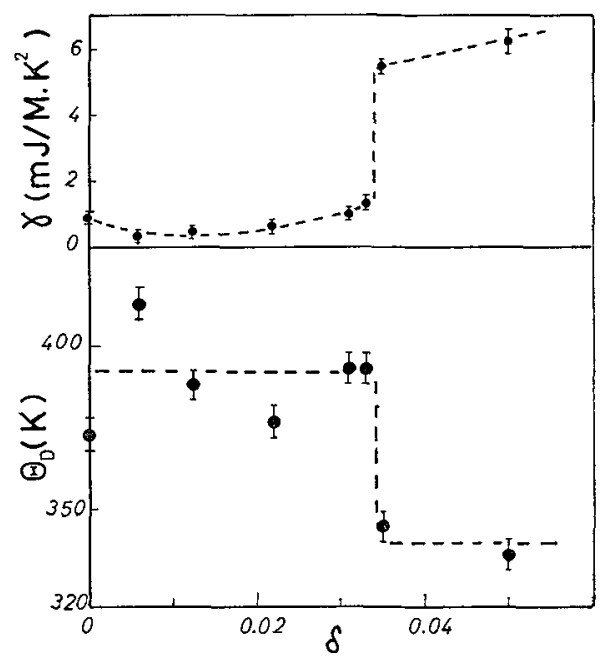

Fig. 7. - Parameters derived from the low-temperature specific heat of $\mathrm{Ni}_{1-\delta S}$. The Debye temperature is deduced from the $T^{3}$ term and $\gamma$ is the coefficient of the term linear in $T$.

density of states is 2.6 states/eV/molecule giving $E_{\mathrm{F}}=1.2 \mathrm{eV}$ on a free electron model. No exact account of the physical properties should be expected of the free electron model when applied to a d-band metal, but the fair agreement among the values of $E_{\mathrm{F}}$ derived from the three different measurements and with the width of the peak in figure $2 b$, all point to a picture of the metallic phase of NiS in which interelectronic correlations are rather small. If we suppose that the Pauli susceptibility is enhanced by a factor $f$ of 3 compared with the true density of states, deduced from $\gamma$, then using the result [18].

$$
\alpha=\frac{f-1}{f}=(U+(m-1) J) \frac{\rho_{b}\left(E_{\mathrm{F}}\right)}{m}
$$

where $\rho_{b}\left(E_{\mathrm{F}}\right)$ is the bare density of states per spin at the Fermi level, $m=2$ for $e_{\mathrm{g}}$ electrons and $J \simeq 0.1 U$, we find $U=0.9 \mathrm{eV}$.

The metallic phase of NiS may be contrasted with that of $\mathrm{V}_{2} \mathrm{O}_{3}$. In $\mathrm{V}_{2} \mathrm{O}_{3}$, there is a large paramagnetic susceptibility which decreases with increasing temperature (Curie-Weiss like), a greatly enhanced electronic specific heat $(\gamma=54 \mathrm{~mJ} / \mathrm{mole} / \mathrm{K})$ and a resistivity which follows a $T^{2}$ law up to $100 \mathrm{~K}[13,17]$. Metallic $\mathrm{V}_{2} \mathrm{O}_{3}$ is a highly correlated metal with $U \simeq 2 \mathrm{eV}$ [18]. Metallic NiS is not.

Another indication of electron correlation in a metal is the appearance of a local moment. For paramagnetic metallic NiS, it was found that the incoherent magnetic neutron scattering was independent of scattering vector $[19,20]$ and an upper limit of $0.5 \mu_{B}$ was deduced for the (disordered) moment on the nickel on the timescale of the neutron experiment, $\simeq 10^{-13} \mathrm{~s}$. However, in X-ray photoemission measurements Hüfner and Werthe1m [21] and the present authors find a broadening of the $\mathrm{Ni} 3$ s line which could be attributed to exchange splitting due to a nickel moment of $1.5 \mu_{\mathrm{B}}$. However, the time scale here is comparable with the time $\left(3 \times 10^{-16} \mathrm{~s}\right)$ associated with the bandwidth via the uncertainty principle, which is the time a «local moment » may be expected to persist in a normal metal.

2.2 Low Temperature Phase $T<T_{\mathrm{t}}$ - The characterization of this phase requires more subtle experimental analysis, for two reasons. Firstly, the large volume change at $T_{\mathrm{t}}$ causes crystals to crack up on passing the transition. Even rather homogeneous tiny crystals exhibit a specific heat anormaly at the transition which is composed of numerous narrow spikes, each associated with the transitions of a domain with slightly different stoichiometry [7]. Precise measurements of any bulk transport property are very difficult on account of the cracking. Secondly, the effects of non-stoichiometry on the transport properties is difficult to evaluate. The resistivity, for instance, is almost independent of temperature below the transition and is neither clearly semiconducting nor metallic. The low temperature specific heat however, shows a small linear contribution comparable to that of copper, $\gamma=0.8 \pm 0.3 \mathrm{~mJ} /$ mole $\mathrm{K}$. Its behaviour as a function of vacancy concentration, shown in figure 7 , suggests that the Fermi level lies just above a minimum in the density of states. 
We believe that the antiferromagnetic phase of NiS is an antiferromagnetic semimetal. In other words, there is a fairly small density of spin polarized d-states at the Fermi level. That NiS orders antiferromagnetically below $T_{\mathrm{t}}$ with a moment close to its value at $T=0$, was first shown by Sparks and Komoto [19]. The experimental evidence that it is an itinerant electron antiferromagnet is summarised below.

1) The spin wave velocity deduced from the dispersion relation is exceptionally high, $\simeq 90 \mathrm{~km} / \mathrm{s}$ [22]. Such high velocities are characteristic of metallic antiferromagnets $[22,23]$. If the spin wave dispersion relations are interpreted in the usual way with an Heisenberg Hamiltonian, unrealistically large values are obtained for the exchange constants and ordering temperature [23].

2) The magnetic susceptibility has the curious feature shown in figure 8 , that $\chi_{\|}$is independent of tempe-

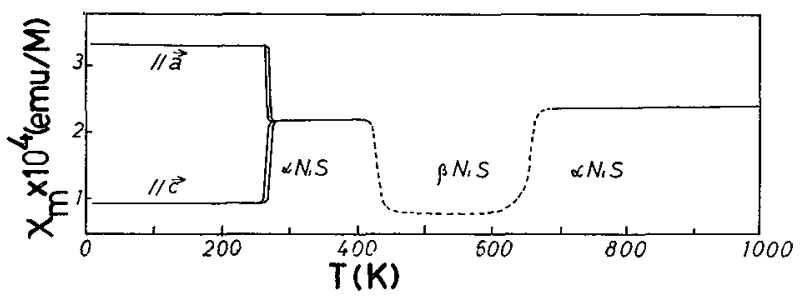

FIG. 8. - Magnetic susceptibility of NiS as a function of temperature. (The NiAs $\alpha$-phase, obtained by quenching from $700 \mathrm{~K}$, transforms to millerite, the $\beta$ phase at about $400 \mathrm{~K}$, but reverts to $\alpha \mathrm{NiS}$ at $650 \mathrm{~K}$.)

rature (the magnetic structure consists of ferromagnetic $c$-plane sheets of nickel whose moments are alternatively parallel and antiparallel to the $c$-axis). Such behaviour is essentially that predicted in a Hubbard model with subbands which overlap at $T=0$ [24]. It may also be obtained in the Stoner-Lidiard model of itinerant electron antiferromagnetism [25].

3) The magnetic hyperfine field on ${ }^{61} \mathrm{Ni}$ nuclei shows a temperature dependence of the form

$$
\left(H_{\mathrm{hf}}(0)-H_{\mathrm{hf}}(T)\right) / H_{\mathrm{hr}}(0)=\alpha T^{2}
$$

where $\alpha \simeq 1-2 \times 10^{-6} / \mathrm{K}^{2}$ depending on stoichiometry [26]. The magnetic excitations are not spin waves at low temperatures because of the gap of $13 \mathrm{meV}$ in the spin-wave dispersion relation. The $T^{2}$ variation may be explained by single-particle magnetic excitations at the Fermi surface [24, 25].

4) The magnetic moment on the nickel at $T=0$ is a very sensitive function of lattice parameter (stoichiometry), decreasing from 1.5 to $1.0 \mu_{\mathrm{B}}$ with a $0.6 \%$ decrease in Ni-Ni distance (Fig. 9). This is also qualitatively what is expected of overlapping Hubbard bands, the Mott-Hubbard splitting collapsing rapidly with decreasing interatomic distance.

The electrical properties of both phases of NiS derive

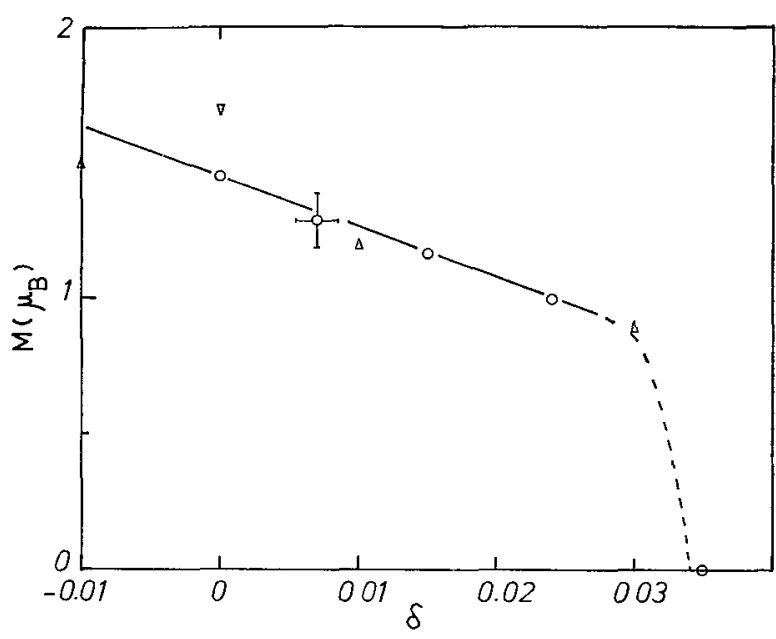

FIG. 9. - The magnetic moment per nickel atom in antiferromagnetic $\mathrm{Ni}_{1-\delta} \mathrm{S}$ measured at $4.2 \mathrm{~K}$ (from ref. [20] and ref therein).

from the presence of $3 \mathrm{~d}(\mathrm{Ni})$-like electrons and $3 \mathrm{p}(\mathrm{S})$ like holes at the Fermi surface, since the two bands are strongly hybridized, as indicated by band structure calculations [27] and suggested by the data of figure 2 . The latter are more mobile, and dominate the Hall effect and thermoelectric power below $T_{t}$ [28]. Above $T_{t}$, the eightfold increase in density of states at the Fermi level is due to the disappearance of the gap which existed in the d-band around most of the Fermi surface.

The picture of the two phases of NiS given in the preceeding paragraphs is essentially the same as that proposed several years ago by White and Mott [29] on the basis of more limited experimental evidence.

3. FeS. - The alpha transition in FeS cannot be completely suppressed by a few percent of iron vacancies. The $T: \delta$ phase diagram is complex and several vacancy superstructures have been reported. A schematic diagram is shown in figure 10 , where the varia-

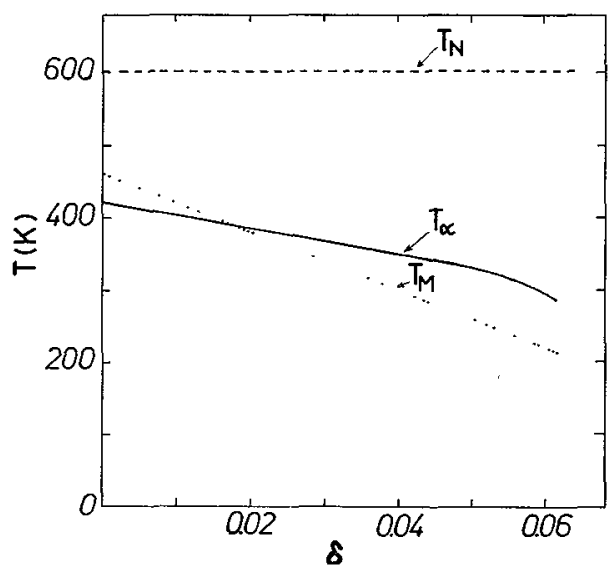

FIG. 10. - Variation of the Néel point, $T_{\mathrm{N}}$, the spin-rotation temperature $T_{M}$ and the alpha transition (taken to be the upper limit of the two-phase region), $T_{\alpha}$ with stoichiometry in $\mathrm{Fe}_{1-\delta} \mathrm{S}$.

The data is taken from references [3], [8] and [31]. 
tion of the Néel point, alpha transition and spin rotation temperature, are given. Unlike NiS, the disappearance of antiferromagnetic order does not coincide with the electronic phase transition.

Extrapolating the reported pressure-dependence of $T_{\alpha}$ [30], a pressure of some $500 \mathrm{kbar}$ would be needed to stabilize the low-temperature phase down to zero temperature. It is therefore impossible to characterize this phase as was done for NiS. Nevertheless, it is stable in a wide range of temperature above $400 \mathrm{~K}$ where its physical properties have been measured.

The samples of $\mathrm{Fe}_{1-\delta} \mathrm{S}$ and $\left(\mathrm{Fe}_{x} \mathrm{Ni}_{1-x}\right)_{1-\delta} \mathrm{S}$ which we have studied were all taken from large single crystals grown by the Bridgmann method.

3.1 High temperature Phase $T>T_{\alpha}$. The phase appears to be a poor, highly-correlated metal. It remains antiferromagnetically ordered up to $600 \mathrm{~K}$ although the exchange interaction deduced from $\chi_{\perp}$ (Fig. 11) [31, 33] or the temperature dependence

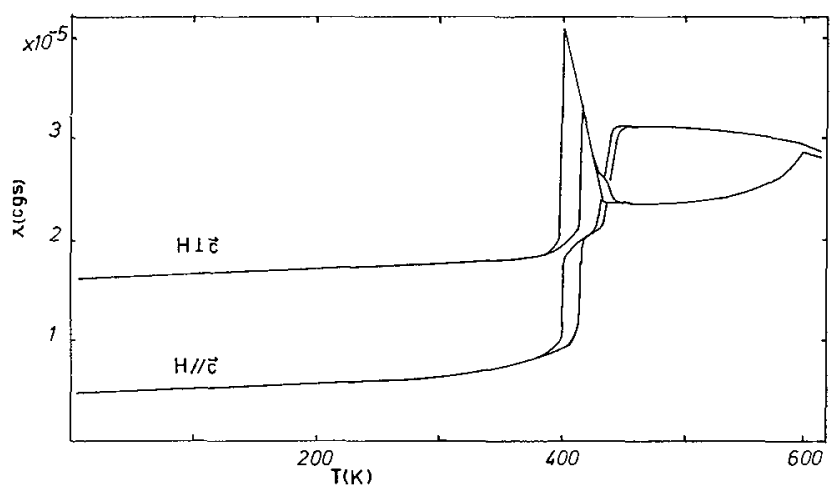

FIG. 11. - Magnetic susceptibility of $\mathrm{Fe}_{0.995} \mathrm{~S}$.

of the ${ }^{57} \mathrm{Fe}$ hyperfine field $[34,38]$ is $30 \%$ less than in the low temperature phase. Even above $T_{\mathrm{N}}$ the susceptibility seems to follow a Curie-Weiss law $\chi_{\mathrm{M}}=C /(T+\theta)$ with $C=3.38$ which gives $P_{\text {eff }}=5.22 \mu_{\mathrm{B}}$ (the expected value for $S=2$ is $4.88 \mu_{\mathrm{B}}$ ) and $\theta \simeq 900 \mathrm{~K}$ [32]. The Morin transition where the spins rotate from $c$ for $T<T_{\mathrm{M}}$ to $\perp c$ for $T>T_{\mathrm{M}}$ has no special bearing on the electronic properties, but it permits an estimate of the single-ion anisotropy if the spin rotation may be explained, as in $\alpha \mathrm{Fe}_{2} \mathrm{O}_{3}$, by a competition of dipolar and single-ion contributions. We estimate $D \simeq 1 \mathrm{~cm}^{-1}$ only, a much smaller value than normal for the $\mathrm{Fe}^{2+}$ ion in a $\mathrm{A}_{1}$ or $\mathrm{E}_{2}$ ground state. Further evidence that the iron is not in a pure crystal field state is provided by the electric field gradient on the ${ }^{57} \mathrm{Fe}$ nucleus. A Mössbauer spectrum taken above $T_{\mathrm{N}}$ (Fig. 12a) gives a quadrupole splitting of only $0.4 \mathrm{~mm} / \mathrm{s}$. The electric field gradient is usually decomposed as a sum of ionic and lattice contributions. The latter rarely exceeds $\pm 0.5 \mathrm{~mm} / \mathrm{s}$, whereas the former has the opposite sign, and is approximately - $3.6 \mathrm{~mm} / \mathrm{s}$ for the $\mathrm{A}_{1}$ state and $+1.8 \mathrm{~mm} / \mathrm{s}$ for the $\mathrm{E}_{2}$ state. It follows that the $" \mathrm{Fe}^{2+}$ ion 》 is in a mixture

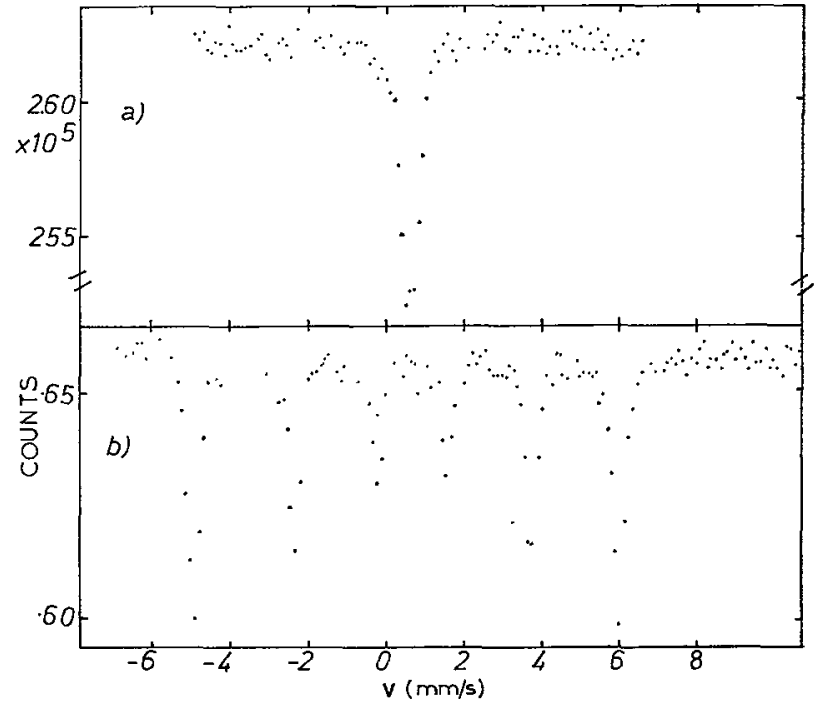

FIG. 12. - $57 \mathrm{Fe}$ Mössbauer spectra of $\mathrm{FeS} a$ ) at $650 \mathrm{~K}$ and b) at $296 \mathrm{~K}$.

of the two. In other words, the sixth $\downarrow$ electron finds itself in a mixture of $t_{2 g}$ orbitals with $a_{1}$ and e character, which suggests that the bandwidth of each of these states is much greater than their separation in the trigonal crystal field $\simeq 0.1 \mathrm{eV}$. The mobility of this electron moving in a narrow spin-polarised band is presumably small and anisotropic, so that the conductivity remains dominated by $3 p(S)$ holes at the Fermi level. There may be polaronic effets in the narrow band, with movement of the triangular $\mathrm{Fe}_{3}$ molecules [4] which were the basis of the low temperature superstructure.

3.2 Low TEMPerature PHASE. - The low temperature structure is a slightly distorted version of the NiAs structure, in which the essential change is the formation of small triangles in the previously hexagonal iron planes (Fig. 13). The new cell is $(\sqrt{3} a, 2 c)$. Goodenough has suggested that the iron $3 \mathrm{~d}$ electrons are partly delocalised in bonds [35]. The conductivity is not typically semiconducting. It increases by only two orders of magnitude from 0.1 to $10 \Omega^{-1} \mathrm{~cm}^{-1}$ between 4.2 and $300 \mathrm{~K}$, and can be fitted neither to a single activation energy nor to $a T^{1 / 4}$ law [36] in this range of temperature. The Seebeck coefficient however, remains p-type. A possible explanation taking an ionic model for FeS is that the iron vacancies are compensated by the appearance of bonded sulphur pairs $\mathrm{S}_{2}^{2-}$ in their vicinity (cf. $\mathrm{FeS}_{2}$ ). These sulphur molecules will act as acceptors near the top of the $3 \mathrm{p}(\mathrm{S})$ valence band, and they are present in such numbers $\sim 10^{20} / \mathrm{cc}$ for $\mathrm{Fe}_{0.996} \mathrm{~S}$, that they should form as impurity band and drain some electrons from the top of the sulphur $3 p$ band. Conduction would be principally by the $3 p$ holes.

It is as yet unresolved whether there are any $3 \mathrm{~d}(\mathrm{Fe})$ states at the Fermi level. The spin-wave velocities are much lower than in NiS [22] and the dispersion rela- 


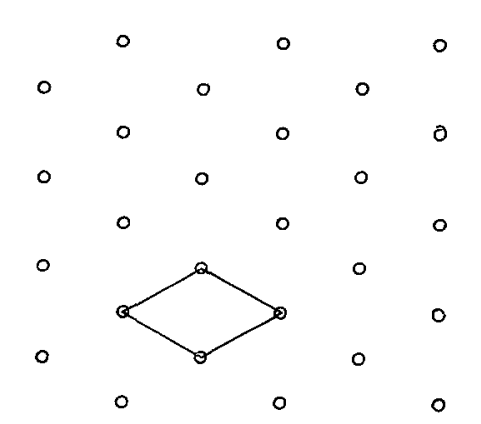

a)
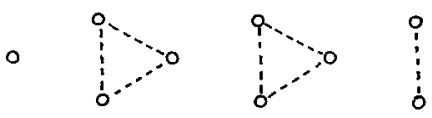

b)

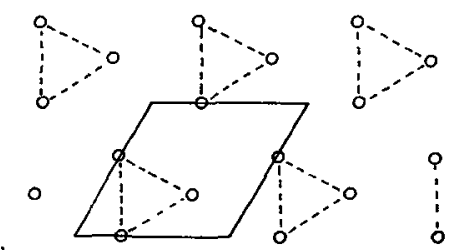

FIG. 13. - Iron planes $a$ ) in the undistorted NiAs structure $T>T_{\alpha}, b$ ) in the distorted structure, $T<T_{\alpha}$. The base of the unit cell is outlined.

tions may be fitted with a normal Heisenberg Hamiltonian. However, $\chi_{\|}$seems to depend sensitively on stoichiometry [31] and may reflect a high density of states in the acceptor impurity band.

4. Origins of the transitions. - Here we consider the question «why do the transitions occur?». The first place to look for an explanation of an electronic phase transition as a function of temperature, would be to the electrons themselves. An increase in the electronic density of states at the Fermi level results in an increase of electronic entropy of order $\gamma T$. One reason why insulator-metal transitions are relatively uncommon is because the appearance of a Fermi surface in a Mott insulator would require a decrease in the interatomic distances to increase $\Delta / U$. This is usually accompanied by an increase in elastic constants and a large reduction of lattice entropy so that the net electronic plus lattice entropy change is unfavourable for the transition to occur. However, in many of the substances where electronic phase transitions do occur as a function of temperature, the lattice seems to be a contributing rather than an impeding factor, possibly because of the electron-phonon interaction.

Our approach to try to understand the origins of the transitions in FeS and NiS consists of analysing the total entropy of the transition $\Delta S_{\text {tot }}$ as the sum of electronic, lattice and magnetic contributions.

Before attempting to evaluate these contributions from the experimental data, we remark that there is as significant difference in the specific heat anomalies themselves for the two sulphides despite the fact that both transitions are first order, and the entropy changes in stoichiometric samples are similar $\Delta S_{\text {tot }}=5.3 \mathrm{~J} /$ mole $\mathrm{K}$ for NiS and $5.9 \mathrm{~J} /$ mole $\mathrm{K}$ for $\mathrm{FeS}$. In NiS, the transition is very sharp as a function of temperature, with a structure in thermal measurements on a tiny crystal $(\simeq \mathrm{lmg}$ ) consisting of a few sharp spikes spaced out over a one or two degrees in temperature [20]. Similar structure was found in the transitions in other compounds such as $\mathrm{V}_{2} \mathrm{O}_{3}$ where the transition temperature is a sensitive function of stoichiometry [37]. It is attributed to the presence of domains of slightly differing stoichiometry. In FeS however, the specific heat anomaly is broadened towards the low temperature side, and shows no sharp structure even in tiny crystals. There is a two phase region several tens of degrees wide where the high temperature phase coexists continuously with the low temperature phase.

4.1 NiS. - The electronic contribution to the entropy may be evaluated from the electronic specific heats of the two phases. The total specific heat at low temperatures is of the form $C_{\mathrm{P}}=\gamma T+\beta T^{3}$; $\Delta S_{\mathrm{el}}=\Delta \gamma T_{\mathrm{t}}$. From the data of figure 7 , the average value of $\gamma$ is $0.7 \mathrm{~mJ} /$ mole $\mathrm{K}^{2}$ for the semimetallic samples and $6.0 \mathrm{~mJ} /$ mole $\mathrm{K}^{2}$ for the metallic ones. It follows that $\Delta S_{\mathrm{el}}=5.3 T_{\mathrm{t}} \mathrm{mJ} / \mathrm{mole} \mathrm{K}$, i. e. $1.4 \mathrm{~J} / \mathrm{mole} / \mathrm{K}$ for stoichiometric NiS, or only a quarter of the total entropy. A similar result is obtained in the change in electronic density of states at $T_{\mathbf{t}}$ is evaluated from the change in Pauli susceptibility

$$
\left(\chi\left(T>T_{\mathrm{t}}\right)-\chi_{1]}\left(T<T_{\mathrm{t}}\right)\right) .
$$

The lattice contribution to the entropy of transition is also positive and actually greater than the electronic contribution. Extensive evidence in support of this contention is provided by four different experiments.

a) The $T^{3}$ terms in the low temperature specific heats for the semimetallic and metallic phases give Debye temperatures of $390 \pm 15 \mathrm{~K}$ and $340 \pm 10 \mathrm{~K}$. Using the Debye model, a lattice entropy difference of $6.25 \pm 2.2 \mathrm{~J} /$ mole $\mathrm{K}$ is obtained at $265 \mathrm{~K}$. However, these temperatures are characteristic of the low frequency accoustic modes only and take no account of higher energy accoustic or optical modes which will be excited at least in the near stoichiometric samples with high transition temperatures.

b) The weighted phonon density of states has been measured directly in the same sample just above and just below $T_{\mathrm{t}}$ by inelastic neutron scattering [39]. These measurements by Gompf et al. were made by a time of flight method and show clearly the softening of accoustic phonons. In addition, there were modifications in the density of states, notably around $20 \mathrm{meV}$ and $35 \mathrm{meV}$. Nevertheless, it is the change in the low energy accoustic phonons which is important for the transition in NiS since some samples have their transition as low as $60 \mathrm{~K}$. In FeS however, the zone boundary 
phonons should play a key role in the structural phase transition at $T_{\alpha}$. Gompf et al. have calculated the specific heat from their density of states measurement [39] which we then used to calculated the change in lattice entropy as a function of transition temperature, as shown in figure 14. The electronic contribution $\Delta S_{\mathrm{el}}$ and the measured total entropy are also shown.

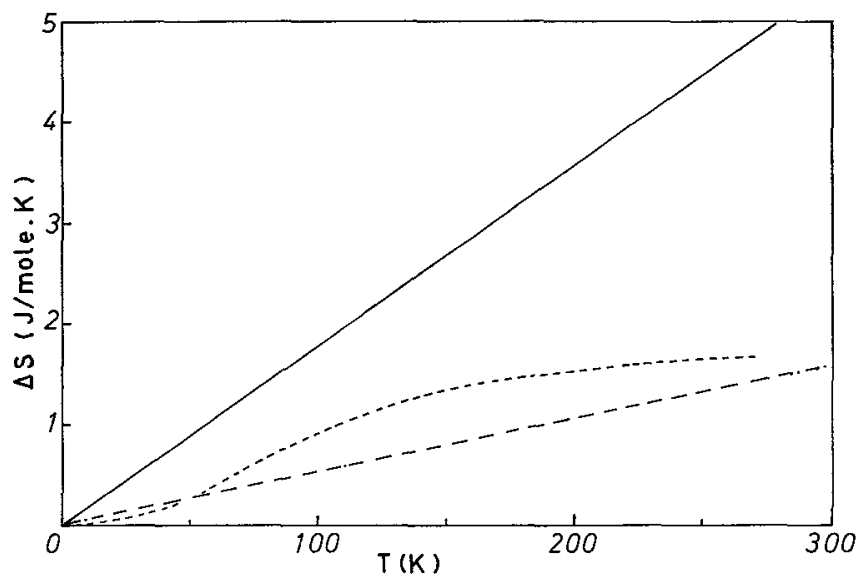

FIG. 14. - Contributions to the entropy change at the transition in $\mathrm{Ni}_{1-\alpha} \mathrm{S}$; — represents the experimentally measured total entropy (data from reference [7]), .-...- represents the electronic contribution, - - - represents the lattice contribution calculated from the phonon density of states (reference [39]).

c) Further evidence of the softening of the accoustic phonons at the transition is provided by preliminary data on the elastic constants derived from the initial slopes of the phonon dispersion relations measured by inelastic neutron scattering [40] $C_{66}$ and in particular $C_{44}$ soften abruptly at the transition but $C_{33}$ and $C_{11}$ remain almost unchanged. The bulk modulus also decreases at the transition [41].

d) Finally, measurements by Fink et al. of the Debye-Waller factor by the Mossbauer effect of ${ }^{61} \mathrm{Ni}$ [26] show a large difference between the semimetallic and the metallic samples. In the case of $\mathrm{Ni}_{0.971} \mathrm{~S}$, there is a $30 \%$ decrease in $f$ at its transition at $T_{\mathrm{t}}=80 \mathrm{~K}$. These authors interpret their data with a Debye plus Einstein model for the phonons, and find that $\theta_{\mathrm{D}}$ for ${ }^{61} \mathrm{Ni}$ decreases from $230 \mathrm{~K}$ to $193 \mathrm{~K}$ on heating through $T_{\mathrm{t}}$.

With all the above evidence, it may be affirmed that there is a significant softening of the accoustic phonons in the metallic phase of NiS and that there is a contribution $\Delta S_{\text {latt }}$ to the entropy of transition which is approximately $2 \mathrm{~J} /$ mole $\mathrm{K}$ in stoichiometric NiS. Detailed agreement of the Debye temperatures derived from different measurements is not to be expected because of the different weighting of the phonon density of states.

A third contribution to the entropy of the transition could be of purely magnetic origin. It is difficult to evaluate but the entropy of the antiferromagnetic phase just below the transition should be small because the moments are within $10 \%$ of their value at $T=0$. The metallic phase, as discussed in section 2.1 , is not highly correlated and there are certainly no local moments on the nickel. If the correlations were negligible, there would be no magnetic entropy in the metal. From our present data, this magnetic contribution may be estimated as $0.25 R \ln 3$.

4.2 FeS. - The experimental data needed to analyse the transition entropy in $\mathrm{FeS}$ is much less abundant than for NiS, and in particular, there is no way as yet to evaluate the electronic contribution.

There have been Mössbauer measurements of the Debye Waller factor of ${ }^{57} \mathrm{Fe}$ in FeS by Thiel and Van Den Berg [34] Townsend et al. [38] and ourselves which can be used to estimate $\Delta S_{\text {latt }}$. The discontinuity decrease of the recoilless fraction at the transition, interpreted on a Debye model, gives an increase in lattice entropy in the high temperature phase of order $2 \mathrm{~J} /$ mole $\mathrm{K}$.

The magnetic contribution to the transition entropy can be simply evaluated on the molecular field model, using the change in $\chi$ or the difference between the temperature derived from extrapolating the hyperfine field for $T>T_{\alpha}$ to zero and $T_{\mathrm{N}}$ to estimate the change in reduced temperature at $T_{\alpha}$. The change is from 0.55 to 0.73 , which corresponds to an entropy increase of $3.2 \mathrm{~J} /$ mole for $S=2$. The molecular field model should be a reasonable approximate for $\mathrm{FeS}$ because the iron has a good local moment, even above $T_{\mathrm{N}}$. Further discussion of the $\alpha$ transition is given in the paper of Townsend et al. [38].

5. Iron impurities in metallic NiS. - The truth of the last sentence is nicely demonstrated by the behaviour of iron impurities in a host of metallic nickel sulphide. We can distinguish two ranges of concentration in $\left(\mathrm{Fe}_{x} \mathrm{Ni}_{1-x}\right)_{1-\delta} S$ with $\delta \simeq 0.04$.

$x \lesssim 0.01$ : At low concentrations, the excess susceptibility due to iron impurities in a host of metallic nickel sulphide follows a Curie-Weiss law with $P_{\text {eff }}=1.2 \mu_{\mathrm{B}}$ /iron and $\theta=7 \mathrm{~K}$, shown in figure 15 . The excess susceptibility scales with the iron concentration up to roughly $1 \%$ [42]. No trace of magnetic order was detected in the Mössbauer spectrum of $\left(\mathrm{Fe}_{0.01} \mathrm{Ni}_{0.99}\right)_{0.948} \mathrm{~S}$ down to $40 \mathrm{mK}$ in measurements made by $\mathbf{P}$. Steiner. The electrical resistivity of the iron-doped samples shows a shallow minimum at a temperature which depends on concentration. The residual resistivity is linear in $x$, and the excess resistivity may be fitted to a relation of the form

$$
\Delta \rho / x=A+B \ln \left[1+\left(T / \theta_{\mathrm{c}}\right)^{2}\right]
$$

with $\theta_{\mathrm{c}} \simeq 40 \mathrm{~K}$. These data are quite suggestive of a Kondo effect.

$0.01<x<0.10:$ At higher concentrations of iron, the behaviour is completely different.

Magnetic order appears at a temperature which depends on both $x$ the iron concentration, and $\delta$, 
the vacancy concentration. Two types of variation of the magnetic susceptibility have been found in different crystals. In the one shown in figure $16 a$, there is an extremely sharp peak in $\chi_{\|}$at $T_{\mathrm{N}}$ and the peak does not

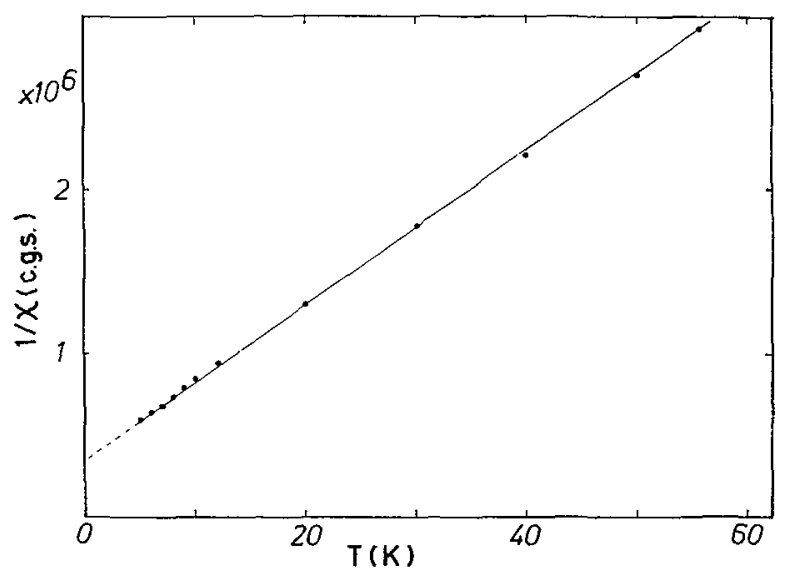

FIG. 15. - Curie-Weiss behaviour of the susceptibility of the iron in $\left(\mathrm{Fe}_{0.01} \mathrm{Ni}_{0.99}\right)_{0.95} \mathrm{~S}$.

flatten out in increasing fields in the range 10010000 Oe. $\chi$ shows a small temperature-dependence below $T_{\mathrm{N}}$, and above $T_{\mathrm{N}}$ there is a Curie-Weiss susceptibility with $P_{\text {eff }}=2.2 \mu_{\mathrm{B}}$ iron and $\theta \simeq 0$. We interpret these data as showing that the nickel has no moments, but that the iron impurities carry welldefined moments which order magnetically. This order may be a dilute antiferromagnetism or else it could be related to the order found in spin glasses like $\mathrm{CuMn}$ or $\left(\mathrm{V}_{x} \mathrm{Ti}_{1-x}\right)_{2} \mathrm{O}_{3}$. There is a very slight thermoremanence of order $3 \times 10^{-4} \mathrm{emu} / \mathrm{g}$ when cooled to $4.2 \mathrm{~K}$ in a field of $10 \mathrm{kOe}$ parallel to the $c$-axis. The invariance of the peak in the susceptibility in different applied fields suggests that the magnetic ordering occurs at a definite phase transition, and it is is conceivable that the framework of the antiferromagnetic structure of NiS or FeS is retained in the dilute alloys i. e. that alternate planes are coupled antiferromagnetically.

The progression from «Kondo-like » to « spinglasslike » magnetic behaviour with increasing concentration of the magnetic species in these dilute $\mathrm{Ni}_{0.95} \mathrm{~S} F$ alloys seems to be rather analogous to the dilute alloys of transition metals in the noble metals. It underscores the difference between the iron and nickel d-electrons.

When the vacancy concentration is reduced a little, a NiS-type transition reappears, as shown in the example of figure $16 b$. The iron noment deduced from the excess Curie-Weiss susceptibility above $T_{\mathrm{t}}$ is $2.2 \mu_{\mathrm{B}}$, and the nickel appears to regain its moment only below $T_{1}$. Like near stoichiometric NiS, the crystal breaks up on cycling the transition.

All these results are summarised in the $T ; \delta ; x$ diagram of figure 17 .

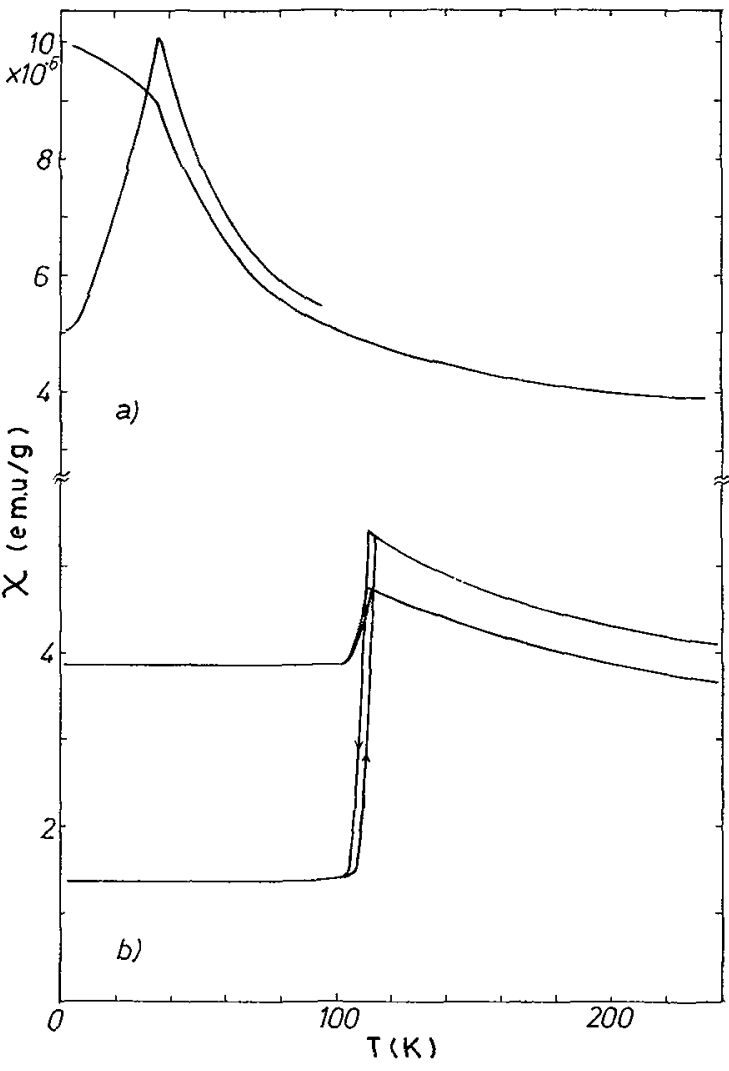

Fig. 16. - Susceptibility of single crystal of a) $\left(\mathrm{Fe}_{0.045} \mathrm{Ni}_{0.955}\right)_{0.951} \mathrm{~S}$ b) $\left(\mathrm{Fe}_{0.05} \mathrm{Ni}_{0.95}\right)_{0.962} \mathrm{~S}$.

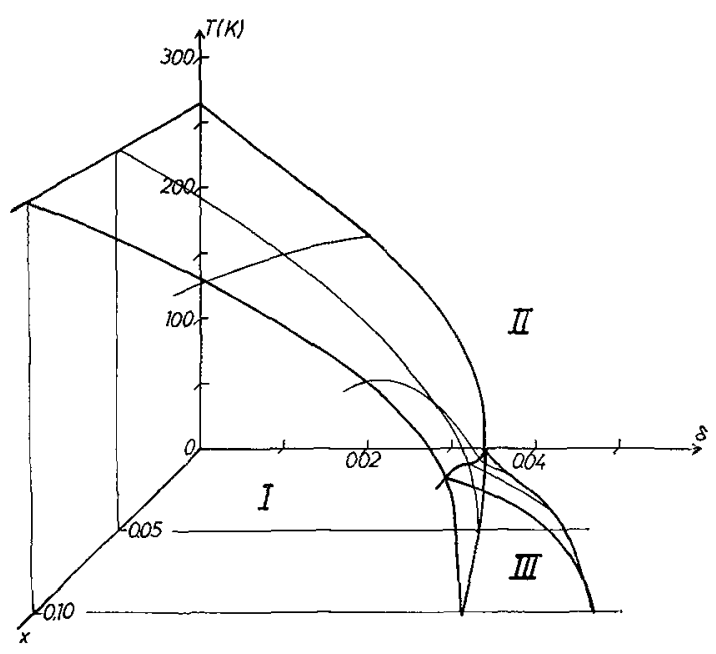

FIG. 17. - Tentative phase diagram for $\left(\mathrm{Fe}_{x} \mathrm{Ni}_{1_{-x}}\right)_{1_{-\alpha} \mathrm{S} \text { showing }}$ three distinct regions :

I. Fe and Ni moments ordered antiferromagnetically,

II. Ni Pauli paramagnetic and Fe Curie-Weiss paramagnetic,

III. Ni Pauli paramagnetic but Fe moments ordered (dilute antiferromagnet or spin glass).

6. Conclusion. - Both the electronic structures of the two phases and the reasons for the phase transition are different in NiS and FeS. The data is fairly complete for $\mathrm{NiS}$ and it is clear that its transition as a function of 
temperature, from an itinerant-electron semimetallic antiferromagnet to a normal weakly correlated metal is not a purely electronic phase transition. The interaction of the electrons with the lattice, particularly the low energy accoustic phonons, is crucial and the increase in lattice entropy at the transition is at least as large as the increase in electronic entropy.

In FeS, the limitcd data available suggest that the transition at $T_{\alpha}$ is from a degenerate semiconductor to a poor metal. Both phaces are antiferromagnetic, but a sharp reduction in exchange at $T_{\alpha}$ provides a large magnetic contribution to the entropy of transition. The lattice entropy also increases considerably, but the electronic contribution cannot yet be evaluated.

The results show that consideration of the electrons alone will not explain the phase transitions. The rough evaluations of the contributions to the transition entropy show which are the important physical factors to be taken into account but do not quite answer the question why the phase transitions occur. The large phonon contribution is probably a rather general factor in metal-nonmetal transitions. In NiS, it seems likely that it is a consequence of the change in electronic structure, through the electron-phonon interaction, whereas in FeS, the change in lattice structure brought about by exciting high energy lattice modes seems to be the key, and the change in electronic structure and exchange the consequences.

The experiments on iron impurities in a host of metallic nickel sulphide illustrate quite vividly the difference in the magnetism of iron and nickel. There is no moment on the nickel, just a weakly correlated d-band with Pauli susceptibility. The iron impurities keep their moment but at low concentrations, there is a Kondo effect. At larger concentrations, the exchange among the iron impurities dominates, and a dilute antiferromagnetic or else a spin-glass-like order appears. Both these effects, well-known for noble metal hosts, have not previously been observed in a host which is a transition-metal compound, except for the spin-glass behaviour of $\left(\mathrm{Ti}_{1-x} \mathrm{~V}_{x}\right)_{2} \mathrm{O}_{3}$ [43].

Acknowledgements. - The authors have greatly benefited from collaboration with many co-workers, particularly G. Czjzek, J. Fink, H. Fuess, F. Gompf, P. Haen, R. Lagnier, G. A. Sawatzky and P. Steiner.

\section{References}

[1] Wells, A. F:, Structural Inorganic Chemistry (Oxford) 1962.

[2] Ohtani, T., J. Phys. Soc. Japan 37 (1974) 701.

[3] Moldenhauer, W. and Bruckner, W., Phys. Stat. Sol. 34 (1976) 565 .

[4] Bertaut, E. F., Bull. Soc. Franc. Miner. Crist. 79 (1956) 293.

[5] MCWhan, D. B., Marezio, M., Remeika, J. P. and DerNIER, P. D., Phys. Rev. B 5 (1972) 2552.

[6] Barthelemy, E., Gorochov, O. and McKinzie, E., Mater. Res. Bull. 8 (1973) 1401

[7] Coey, J. M. D. and Brusetti, R., Phys. Rev. B 11 (1975) 671.

[8] THIEL, R., Thesis, Groningen (1969) (unpublished).

[9] SParks, J. T., Komoto, T., J. Appl. Phys. 34 (1963) 1191.

[10] Haraldsen, H., Z. Anorg. Allgem. Chem. 246 (1941) 195.

[11] Fourcaudot, G., Brusetti, R., Mercier, J., J. Cryst. Growth (in press).

[12] Coex, J. M. D., Roux-Bursson, H. and Chamberod, A., Solid State Commun. 13 (1973) 43.

[13] McWhan, D. B., RemetKa, J. P., Maita J. P., OkinaKa, M., Kosuge, K. and KaCHI, S., Phys. Rev. B 7 (1973) 326.

[14] Koenler, R. F. and Whirte, R. L., J. Appl. Phys. 44 (1973) 1682.

[15] Coey, J. M. D., Bruyere, J. C., Roux-Buisson, H. and BrusstTI, R., Proceedings of the International Conference on Magnetism, Moscow 1973, Vol. IV, p. 166 (Nauka, Moscow, 1974).

[16] Barnard, R. D., Thermoelectricity in Metals and Alloys (Taylor \& Francis, London) 1972 p. 58

[17] MCWhan, D. B. and RiCE, T. M. Phys. Rev. Lett. 22 (1969) 887.

[18] Castellani, C., Natoli, C. R. and Ranninger, J., J. Physique Colloq. 37 (1976) C4-199.

[19] Sparks, J. T. and Комото, T., Rev. Mod. Phys. 40 (1968) 752.

[20] Coey, J. M. D., Brusetti, R., Kallel, A., Schweizer, J. and Fuess, H., Phys. Rev. Leit. 32 (1974) 1257.

[21] Hufner, S. and Wertheim, G. K., Phys. Lett. 44A (1973) 133.

[22] Lowde, R. and PARIsot, G. (private communication).
[23] Briggs, G. A., Duffill, C., Hutchings, M. T., Lowde, R, D., SATya-Murthy, J. S., SAunderson, D. H., StringFELLOW, M. W., WAEBER, W. B. and WINDSOR, C. G. in Neutron Inelastic Scattering (IAEA Vienna) p. 669.

[24] Brandt, U. and Gross, W., Z. Phys. 263 (1973) 69.

[25] Lidiard, A. B., Proc. R. Soc. A 224 (1954) 161.

[26] Fink, J., Czjzek, G., Schmidt, H., Ruebenbauer, K., Coey, J. M. D. and Brusetti, R., J. Physique Colloq. 35 (1974) C6-675.

[27] Mattheiss, L. F., Phys. Rev. B 10 (1974) 995.

[28] Ohtani, T., J. Phys. Soc. Japan 37 (1974) 701.

[29] White, R. M. and Mort, N. F., Phil. Mag. 24 (1971) 845.

[30] Ozawa, K. and AnzaI, S, Phys. Stat. Sol. 17 (1966) 697.

[31] Horwood, J. L., Townsend, M. G. and Webster, A. H., J. Solid State Chem. 17 (1976) 35.

[32] Hirahara, E. and Murakami, M., J. Phys. Chem. Solids, 7 (1958) 281.

[33] Takahashi, T., Solid State Commun. 13 (1973) 1335.

[34] Thiel, R. C. and van Den Berg, C. B., Phys. Stat. Sol. 29 (1968) 837

[35] GoOdenough J. B., Colloque International du C. N. R. S. No 157 (C. N. R. S. Paris) 1965, p. 263 and Mater. Res. Bull. 6 (1971) 967.

[36] Motr, N. F., Metal-Insulator Ttansitions (Taylor and Francis, London) 1974.

[37] Coey, J. M. D., Roux'-Butsson, H., SChlenker, C., LaKkis, S. and Dumas, J., Rev. Gen. Therm. (in press).

[38] Townsend, M. G., Gosselin, J. R., Tremblay, R. J. and Webster, A. M., J. Physique Colloq. 37 (1976) C4-11.

[39] GompF, F., CzJzek, G. and Fink, J., Report KFK 2183, Kernforschungzentrum Karlsruhe, p. 38 (1975).

[40] Parisot, G., Coey, J. M. D., Brusetti, R., Gompf, F., Czjzek, G. and Fink, J., A. I. P. Conf. Proc. 29 (1975) 407.

[41] Smith, E. A. and Sparks, J. T., J. Appl. Phys. 40 (1969) 1332.

[42] Roux-Buisson, H., Coex, J. M. D. and Haen, P., A. $I$. $P$. Conf. Proc. 24 (1974) 57.

[43] Schlenker, C. and Dumas, J., J. Physique Colloq. 37 (1976) C4-41.

[44] Ward, J. C., Rev. Pure Appl. Chem. 20 (1970) 175 